\title{
GENERALIZED MULTI-PEG TOWER OF HANOI PROBLEM
}

\author{
A. A. K. MAJUMDAR ${ }^{1}$ \\ (Received 25 May 1994; revised 28 August 1994)
}

\begin{abstract}
This paper treats the multi-peg generalization of the Tower of Hanoi problem with $n(\geq 1)$ disks and $p(\geq 3)$ pegs, $P_{1}, P_{2}, \ldots, P_{p}$. Denoting by $M(n, p)$ the presumed minimum number of moves required to transfer the tower of $n$ disks from the source peg, $P_{1}$, to the destination peg, $P_{p}$, under the condition that each move transfers the topmost disk from one peg to another such that no disk is ever placed on top of a smaller one, the Dynamic Programming technique has been employed to find the optimality equation satisfied by $M(n, p)$. Though an explicit expression for $M(n, p)$ is given, no explicit expressions for the partition numbers (at which $M(n, p)$ is attained) are available in the literature for $p \geq 5$. The values of the partition numbers have been given in this paper.
\end{abstract}

\section{Introduction}

The classical Tower of Hanoi problem reads as follows.

Three pegs, $P_{1}, P_{2}$ and $P_{3}$, are fastened to a stand, and $n(\geq 1)$ disks of different radii, each with a hole through which a peg can pass, initially rest on the source peg, $P_{1}$, in a tower in small-on-large ordering (with the largest at the bottom, the second largest above it, and so on, with the smallest at the top). The objective is to transfer the tower from $P_{1}$ to the destination peg, $P_{3}$, in the minimum number of legal moves, where each legal move can transfer the topmost disk from any peg to another such that no disk is ever placed on top of a smaller one.

The above problem was first formulated by the French mathematican, Francois Edouard Anatole Lucas in 1883, a biography of whom is given in Hinz [9]. The problem appears in Ball [2] and Gardner [7] as one of mathematical recreation, and in Papadimitriou and Steiglitz [19] as an illustration of the combinatorial optimization problem. Recently, the problem has also drawn the attention of the computer scientists, and it serves as a popular example to illustrate recursion versus iteration in elementary texts (see, for example, Reingold and Hansen [21] and Roberts [22]), and in discrete

\footnotetext{
'Department of Mathematics, Jahangirnagar University, Savar, Dhaka 1342, Bangladesh

(C) Australian Mathematical Society, 1996, Serial-fee code 0334-2700/96
} 
mathematics courses (see, for example, Johnsonbaugh [12]). The problem has been studied by Sohn and Gaudiot $[26,27]$ and Osato [18] in connection with artificial intelligence and robot control. Schmajuk and Thieme [25] and Daum et al. [5] demonstrate the application of their neural network models to the Tower of Hanoi puzzle.

During the last two decades, the classical Tower of Hanoi problem has seen many variations and generalizations in different directions, some of which have been reviewed and posed by Atkinson [1], Wood [30], Walsh [29], Newman-Wolfe [17], Chan [3], Hinz [10, 11], Wu and Chen [31, 32], Zanten [33] and Poole [20].

One natural generalization is the multi-peg case with $p(\geq 4)$ pegs, $P_{1}, P_{2}, \ldots, P_{p}$. This generalization was first proposed by Lucas in 1889 . Later, it was revived by Stewart [28].

The Dynamic Programming (DP) technique has been employed by Wood [30], Roth [24], Hinz [8-10], van de Liefvoort [13, 14], Chu and Johnsonbaugh [4] and Majumdar [16]. Wood considers only the 3-peg problem, and the remaining papers deal with the 4-peg problem.

This paper treats the DP approach of the multi-peg Tower of Hanoi problem. The DP formulation is given in Section 2. In Section 3, we solve the multi-peg problem, giving explicit expressions for the "minimum partition numbers".

\section{Dynamic programming formulation}

Let $M(n, p)$ denote the minimum number of (legal) moves required to solve the Tower of Hanoi problem with $n(\geq 1)$ disks and $p(\geq 3)$ pegs. Then, the DP equation satisfied by $M(n, p)$ is (see, for example, Majumdar [16])

$$
\begin{aligned}
& M(n, 3)=2 M(n-1,3)+1, \quad n \geq 2 \\
& M(n, p)=\min _{0 \leq k \leq n-1}\{2 M(k, p)+M(n-k, p-1)\} ; \quad n \geq 2, p \geq 4,
\end{aligned}
$$

where the trivial boundary conditions are

$$
M(0, p)=0, M(1, p)=1 \text { for all } p \geq 3 .
$$

We recall that the DP equation (2.2) is obtained in the following manner. For $p \geq 4$, the transfer of the tower from $P_{1}$ to $P_{p}$ may be effected in the following three steps :

1. Move optimally the topmost $k$ (smallest) disks from $P_{1}$ to some intermediate peg, using all the pegs, in $M(k, p)$ number of moves.

2. Transfer the remaining $(n-k)$ largest disks on $P_{1}$ to $P_{p}$, using the $(p-1)$ pegs available (the intermediate peg cannot be used by the condition of the problem) in the optimal way in $M(n-k, p-1)$ number of moves. 
3. Shift optimally the $k$ disks from the intermediate peg to $P_{p}$, using all the $p$ pegs, again in $M(k, p)$ number of moves.

Then, $k$ is to be determined so as to minimize the total number of minimum moves in the above three steps. For the 3-peg problem, we must have $k=n-1$, giving the DP equation (2.1).

For the 3-peg Tower of Hanoi problem, Wood [30] has shown that the policy leading to the DP equation (2.1) is indeed optimal. For the generalized $p$-peg problem with $p \geq 4$, it still remains to establish that the policy adopted to derive the DP equation (2.2) is optimal. The only thing in this respect is to prove that the $k$ disks stored on the intermediate peg must be smallest consecutive ones (see, for example, Lunnon [15]). The problem still remains open and challenging. Thus, following Hinz [8], $M(n, p)$ is in fact, the pms (presumed minimum solution) corresponding to the multi-peg case considered in this paper.

It is well-known (see, for example, Hinz [8]) that, for the p-peg problem with $p \geq 4$, the value of $k$ minimizing the right-hand expression inside the braces of (2.2) is not unique. Taking this into consideration, we define the following :

$$
\begin{gathered}
F(n, k, p)=2 M(k, p)+M(n-k, p-1) ; 0 \leq k \leq n-1, n \geq 1, p \geq 4, \\
k(n, p)=\min \{k: M(n, p)=F(n, k, p)\} ; n \geq 1, p \geq 4 \\
K(n, p)=\max \{k: M(n, p)=F(n, k, p)\} ; \quad n \geq 1, p \geq 4
\end{gathered}
$$

where, evidently

$$
k(0, p)=K(0, p)=0, \quad k(1, p)=K(1, p)=0 \text { for all } p \geq 4 .
$$

Thus, for $n \geq 1$ and $p \geq 4$ fixed, $k(n, p)$ and $K(n, p)$ denote respectively the minimum and maximum $k$ at which $F(n, k, p)$ is minimized, and would be called the smallest partition number and the largest partition number respectively. Clearly, for $n \geq 1$ and $p \geq 4$ fixed, $F(n, k, p)$ is uniquely minimized if and only if $k(n, p)=$ $K(n, p)$.

The following results have been established by Majumdar [16].

LEMMA 2.1. For $n \geq 1$ and $p \geq 4$ fixed,

(1) $F(n, k, p)$ is minimized for all $k$ with $k(n, p) \leq k \leq K(n, p)$,

(2) $L(n, p) \leq L(n+1, p) \leq L(n, p)+1(L(n, p)=k(n, p)$ or $K(n, p))$.

The solution to the 3-peg problem (given by the DP equation (2.1)) is

$$
M(n, 3)=2^{n}-1, k(n, 3)=K(n, 3)=n-1 \text { for all } n \geq 1 .
$$




\section{3. $p$-peg Tower of Hanoi problem with $p \geq 4$}

The complete solution to the 4-peg Tower of Hanoi problem, giving explicit expressions for $k(n, 4), K(n, 4)$ and $M(n, 4)$, due to Majumdar [16], is reproduced in the following lemma for future reference.

LEMMA 3.1. For any $r \in\{1,2, \ldots\}$,

(1) $F(r(r+1) / 2, k, 4)$ is uniquely minimized at $k=r(r-1) / 2$,

(2) for $n$ with $r(r+1) / 2<n<(r+1)(r+2) / 2$,

$$
k(n, 4)=n-r-1, \quad K(n, 4)=n-r
$$

(3) for $n$ satisfying $r(r+1) / 2 \leq n \leq(r+1)(r+2) / 2$,

$$
M(n, 4)=(n-r(r-1) / 2-1) 2^{r}+1 .
$$

In the following theorem, we generalize the above results to the multi-peg Tower of Hanoi problem.

THEOREM 3.1. For any $r \in\{1,2, \ldots\}$,

(1) $F\left(\left(\begin{array}{c}p+r-3 \\ p-2\end{array}\right), k, p\right)$ is uniquely minimized at $k=\left(\begin{array}{c}p+r-4 \\ p-2\end{array}\right)$ for all $p \geq 4\left(\right.$ with the convention that $\left(\begin{array}{l}M \\ N\end{array}\right)=0$ if $\left.M<N\right)$,

(2) for $n$ satisfying $\left(\begin{array}{c}p+r-3 \\ p-2\end{array}\right) \leq n<\left(\begin{array}{c}p+r-2 \\ p-2\end{array}\right)$,

$$
M(n+1, p)-M(n, p)=2^{r},
$$

$$
M(n, p)=\sum_{i=0}^{r-1} 2^{t}\left(\begin{array}{c}
p+t-3 \\
p-3
\end{array}\right)+2^{r}\left\{n-\left(\begin{array}{c}
p+r-3 \\
p-2
\end{array}\right)\right\}
$$

(c) $k(n, p)= \begin{cases}\left(\begin{array}{c}p+r-4 \\ p-2\end{array}\right), & \text { if } 0 \leq n-\left(\begin{array}{c}p+r-3 \\ p-2\end{array}\right) \leq\left(\begin{array}{c}p+r-4 \\ p-4\end{array}\right) \\ \left(\begin{array}{c}p+r-4 \\ p-2\end{array}\right)+l, & \text { if } n-\left(\begin{array}{c}p+r-3 \\ p-2\end{array}\right)=\left(\begin{array}{c}p+r-4 \\ p-4\end{array}\right)+l\end{cases}$ where $l \in\left\{1,2, \ldots,\left(\begin{array}{c}p+r-4 \\ p-3\end{array}\right)\right\}$, 
(d) $K(n, p)=\left\{\begin{aligned}\left(\begin{array}{c}p+r-4 \\ p-2\end{array}\right)+m, & \text { if } n-\left(\begin{array}{c}p+r-3 \\ p-2\end{array}\right)=m \\ \left(\begin{array}{c}p+r-3 \\ p-2\end{array}\right), & \text { if }\left(\begin{array}{c}p+r-4 \\ p-3\end{array}\right) \leq n-\left(\begin{array}{c}p+r-3 \\ p-2\end{array}\right) \\ & \leq\left(\begin{array}{c}p+r-3 \\ p-3\end{array}\right)\end{aligned}\right.$

where $m \in\left\{1,2, \ldots,\left(\begin{array}{c}p+r-4 \\ p-3\end{array}\right)\right\}$.

PROOF. Part (1) of the theorem has been pointed out by Rohl and Gedeon [23], and the first two results of part (2) have been established by Frame [6]. So, it only remains to prove parts (c) and (d). The proofs are similar, and we prove part (c) only.

The proof is by double induction on $n$ and $p$. For $p=4$, the validity of the result for all $n \geq 1$ follows from Lemma 3.1. For $n=1$, we have $r=1$ for all $p \geq 4$, and the result holds true.

So, let $r \in\{1,2, \ldots\}$ be such that

$$
\left(\begin{array}{c}
p+r-3 \\
p-2
\end{array}\right)<n+1<\left(\begin{array}{c}
p+r-2 \\
p-2
\end{array}\right)
$$

so that

$$
\left(\begin{array}{c}
p+r-4 \\
p-3
\end{array}\right)<n+1-\left(\begin{array}{c}
p+r-4 \\
p-2
\end{array}\right) \leq\left(\begin{array}{c}
p+r-3 \\
p-3
\end{array}\right)+\left(\begin{array}{c}
p+r-4 \\
p-3
\end{array}\right) .
$$

We now consider the following two cases separately.

Case $1\left(\begin{array}{c}p+r-4 \\ p-3\end{array}\right)<n+1-\left(\begin{array}{c}p+r-4 \\ p-2\end{array}\right) \leq\left(\begin{array}{c}p+r-3 \\ p-3\end{array}\right)$.

In this case, by the induction hypothesis,

$$
\begin{aligned}
2 M( & \left.\left(\begin{array}{c}
p+r-4 \\
p-2
\end{array}\right), p\right)+M\left(n+1-\left(\begin{array}{c}
p+r-4 \\
p-2
\end{array}\right), p-1\right) \\
\quad= & \sum_{t=0}^{r-2} 2^{t+1}\left(\begin{array}{c}
p+t-3 \\
p-3
\end{array}\right)+\sum_{t=0}^{r-1} 2^{t}\left(\begin{array}{c}
p+t-4 \\
p-4
\end{array}\right) \\
& +2^{r}\left\{n+1-\left(\begin{array}{c}
p+r-4 \\
p-2
\end{array}\right)-\left(\begin{array}{c}
p+r-4 \\
p-3
\end{array}\right)\right\} \\
= & 1+\sum_{t=1}^{r-1} 2^{t}\left\{\left(\begin{array}{c}
p+t-4 \\
p-3
\end{array}\right)+\left(\begin{array}{c}
p+t-4 \\
p-4
\end{array}\right)\right\}+2^{r}\left\{n+1-\left(\begin{array}{c}
p+r-3 \\
p-2
\end{array}\right)\right\} \\
= & \sum_{t=0}^{r-1} 2^{t}\left(\begin{array}{c}
p+t-3 \\
p-3
\end{array}\right)+2^{r}\left\{n+1-\left(\begin{array}{c}
p+r-3 \\
p-2
\end{array}\right)\right\} .
\end{aligned}
$$


Also, by part (2) of Lemma $2.1, k(n+1, p)=\left(\begin{array}{c}p+r-4 \\ p-2\end{array}\right)$.

Case 2 $\left(\begin{array}{c}p+r-3 \\ p-3\end{array}\right)<n+1-\left(\begin{array}{c}p+r-4 \\ p-2\end{array}\right) \leq\left(\begin{array}{c}p+r-3 \\ p-3\end{array}\right)+\left(\begin{array}{c}p+r-4 \\ p-3\end{array}\right)$.

Here, letting

$$
n+1-\left(\begin{array}{c}
p+r-4 \\
p-2
\end{array}\right)=\left(\begin{array}{c}
p+r-3 \\
p-3
\end{array}\right)+l, \quad l \in\left\{2,3, \ldots,\left(\begin{array}{c}
p+r-4 \\
p-3
\end{array}\right)\right\},
$$

and noting that,

$$
\left(\begin{array}{c}
p+r-4 \\
p-2
\end{array}\right)<\left(\begin{array}{c}
p+r-4 \\
p-2
\end{array}\right)+l \leq\left(\begin{array}{c}
p+r-4 \\
p-2
\end{array}\right)+\left(\begin{array}{c}
p+r-4 \\
p-3
\end{array}\right)=\left(\begin{array}{c}
p+r-3 \\
p-2
\end{array}\right)
$$

we get, by virtue of the induction hypothesis,

$$
\begin{aligned}
2 M( & \left.\left(\begin{array}{c}
p+r-4 \\
p-2
\end{array}\right)+l, p\right)+M\left(\left(\begin{array}{c}
p+r-3 \\
p-3
\end{array}\right), p-1\right) \\
\quad= & 2\left\{\sum_{t=0}^{r-2} 2^{\imath}\left(\begin{array}{c}
p+t-3 \\
p-3
\end{array}\right)+l 2^{r-1}\right\}+\sum_{t=0}^{r} 2^{t}\left(\begin{array}{c}
p+t-4 \\
p-4
\end{array}\right) \\
= & \sum_{t=0}^{r-1} 2^{t}\left(\begin{array}{c}
p+t-3 \\
p-3
\end{array}\right) \\
& +2^{r}\left\{n+1-\left(\begin{array}{c}
p+r-4 \\
p-2
\end{array}\right)-\left(\begin{array}{c}
p+r-3 \\
p-3
\end{array}\right)+\left(\begin{array}{c}
p+r-4 \\
p-4
\end{array}\right)\right\} \\
= & \sum_{t=0}^{r-1} 2^{\imath}\left(\begin{array}{c}
p+t-3 \\
p-3
\end{array}\right)+2^{r}\left\{n+1-\left(\begin{array}{c}
p+r-3 \\
p-2
\end{array}\right)\right\} .
\end{aligned}
$$

It now remains to show that

$$
\begin{gathered}
k\left(\left(\begin{array}{c}
p+r-3 \\
p-2
\end{array}\right)+\left(\begin{array}{c}
p+r-4 \\
p-4
\end{array}\right)+l, p\right)=\left(\begin{array}{c}
p+r-4 \\
p-2
\end{array}\right)+l \\
l \in\left\{1,2, \ldots,\left(\begin{array}{c}
p+r-4 \\
p-3
\end{array}\right)\right\} .
\end{gathered}
$$


We first show that $F\left(\left(\begin{array}{c}p+r-3 \\ p-2\end{array}\right)+\left(\begin{array}{c}p+r-4 \\ p-4\end{array}\right)+l, k, p\right)$ is not minimized at $k=\left(\begin{array}{c}p+r-4 \\ p-2\end{array}\right)$, for otherwise,

$$
\begin{aligned}
& M\left(\left(\begin{array}{c}
p+r-3 \\
p-2
\end{array}\right)+\left(\begin{array}{c}
p+r-4 \\
p-4
\end{array}\right)+1, p\right)-M\left(\left(\begin{array}{c}
p+r-3 \\
p-2
\end{array}\right)+\left(\begin{array}{c}
p+r-4 \\
p-4
\end{array}\right), p\right) \\
& \quad=M\left(\left(\begin{array}{c}
p+r-3 \\
p-3
\end{array}\right)+1, p-1\right)-M\left(\left(\begin{array}{c}
p+r-3 \\
p-3
\end{array}\right), p-1\right) \\
& \quad=2^{r+1}, \text { by part 2(a) of the theorem. }
\end{aligned}
$$

But, this would violate part 2(a) of the theorem, since

$$
\left(\begin{array}{c}
p+r-3 \\
p-2
\end{array}\right)+\left(\begin{array}{c}
p+r-4 \\
p-4
\end{array}\right)+1<\left(\begin{array}{c}
p+r-2 \\
p-2
\end{array}\right) .
$$

Hence, by Lemma 2.1(2), we must have

$$
k\left(\left(\begin{array}{c}
p+r-3 \\
p-2
\end{array}\right)+\left(\begin{array}{c}
p+r-4 \\
p-4
\end{array}\right)+1, p\right)=\left(\begin{array}{c}
p+r-4 \\
p-2
\end{array}\right)+1 .
$$

Proceeding in this way, we can prove (d). This completes the proof of the theorem.

In passing, we mention that Theorem 3.1 is valid for $p=3$ also.

\section{References}

[1] M. D. Atkinson, "The cyclic Towers of Hanoi", Inform. Proc. Letters 13 (1981) 118-119.

[2] W. W. R. Ball, Mathematical recreations and essays (Macmillan Book Co. Ltd., London, 1959).

[3] T. Chan, "A statistical analysis of the Tower of Hanoi problem", Intrn. J. Computer Math. 28 (1989) 57-65.

[4] I-Ping Chu and R. Johnsonbaugh, "The four peg Tower of Hanoi puzzle", SIGCSE Bulletin 23 (1991) 2-4.

[5] I. Daum, H. Ackermann, M. M. Schugens, C. Reingold, J. Dichgans and N. Birbaumer, "The cerebellum and cognitive functions in humans", Behavioral Neuroscience 107 (1993) 411-419.

[6] J. S. Frame, "Solution to AMM problem 3918", Amer. Math. Monthly 48 (1941) 216-219.

[7] M. Gardner, Mathematical Puzzles and Diversions (Penguin Book, London, 1956).

[8] A. M. Hinz, "An iterative algorithm for the Tower of Hanoi with four pegs", Computing 42 (1989) $133-140$.

[9] A. M. Hinz, "Pascal's Triangle and the Tower of Hanoi", Amer. Math. Monthly 99 (1992) 538-544.

[10] A. M. Hinz, "The Tower of Hanoi", L'Enseignement Math. 35 (1989) 289-321.

[11] A. M. Hinz, "Shortest paths between regular states of the Tower of Hanoi", Inform Sci. 63 (1992) 173-181. 
[12] R. Johnsonbaugh, Discrete Mathematics (Macmillan, New York,1990).

[13] A. van de Liefvoort, "An iterative solution to the four-peg Tower of Hanoi problem", Proc. CSC (1990) 70-75.

[14] A. van de Liefvoort, "An iterative Algorithm for the Reve's puzzle", Computer J. 35 (1992) 91-92.

[15] W. F. Lunnon, "The Reve's puzzle", Computer J. 29 (1986) 478.

[16] A. A. K. Majumdar, "The gereralized four-peg Tower of Hanoi problem", Optimization 29 (1994) 349-360.

[17] R. Newman-Wolfe, "Observations on multi-peg Towers of Hanoi", Rochester Univ. Computer Sci. Dept. Rep. 187 (1986) 1-19.

[18] N. Osato, "MARC : A multiagent robot control system", Systems Comp. Japan 24 (1993) 42-51.

[19] C. H. Papadimitriou and K. Steiglitz, Combinatorial Optimization (Prentice-Hall, New Jersy, 1982).

[20] D. Poole, "The bottleneck Tower of Hanoi problem", J. Recreational Math. 24 (1992) 203-207.

[21] E. M. Reingold and W. J. Hansen, Data Structures (Little, Brown \& Co., New York, 1983).

[22] E. Roberts, Thinking Recursively (John Wiley \& Sons, New York, 1986).

[23] J. S. Rohl and T. D. Gedeon, "The Reve's puzzle", Computer J. 29 (1986) 187-188.

[24] T. Roth, "The Tower of Brahma revisited", J. Recreational Math. 14 (1974) 116-119.

[25] N. A. Schmajuk and A. D. Thieme, "Purposive behavior and cognitive mapping- A neural network model", Biolog. Cybern. 67 (1992) 165-172.

[26] A. Sohn and J. L. Gaudiot, "A connectionist approach to learning legal moves in Tower of Hanoi", Proc. 2nd IEEE Conf. A.I. (1990) 336-371.

[27] A. Sohn and J. L. Gaudiot, "Learning legal moves in planning problems- A connectionist approach", Engg. Appl. Artf. Int. 5 (1992) 239-245.

[28] B. M. Stewart, "Problem 3918", Amer. Math. Monthly 46 (1939) 363.

[29] T. R. Walsh, "The Towers of Hanoi revisited : Moving the rings by counting the moves", Inform. Proc. Letters 15 (1982) 64-67.

[30] D. Wood, "The Towers of Brahma and Hanoi revisited", J. Recreational Math. 14 (1981-82) 17-24.

[31] J. S. Wu and R. J. Chen, "The Tower of Hanoi problem with parallel moves", Inform. Proc. Letters 44 (1992) 241-243.

[32] J. S. Wu and R. J. Chen, "The Tower of Hanoi problems with cyclic parallel moves", Inform. Proc. Letters 46 (1993) 1-6.

[33] A. J. van Zanten, "An iterative optimal algorithm for the generalized Tower of Hanoi problem", Internl. J. Computer Math. 39 (1991) 163-168. 